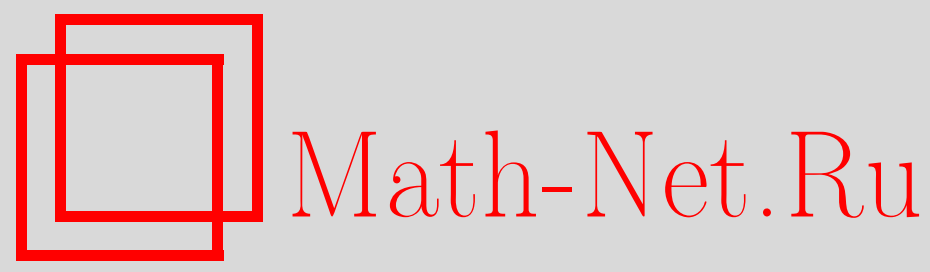

Е. Е. Гуревский, В. А. Емеличев, О пяти типах устойчивости лексикографического варианта комбинаторной задачи на узкие места, Дискрет. матем., 2009, том 21, выпуск 3, 313

DOI: https://doi.org/10.4213/dm1056

Использование Общероссийского математического портала Math-Net.Ru подразумевает, что вы прочитали и согласны с пользовательским соглашением http://www.mathnet.ru/rus/agreement

Параметры загрузки:

IP: 52.6 .47 .48

26 апреля 2023 г., 12:33:43 


\title{
О пяти типах устойчивости лексикографического варианта комбинаторной задачи на узкие места
}

\author{
() 2009 г. Е. Е. Гуревский, В. А. Емеличев
}

\begin{abstract}
Рассматривается векторная комбинаторная минимаксная задача с упорядоченными критериями. Формулируются и доказываются необходимые и достаточные условия пяти известных типов устойчивости задачи, по-разному описывающих поведение лексикографического множества относительно возмущений исходных данных векторного критерия.
\end{abstract}

\section{1. Введение}

Один из известных подходов к исследованию проблем устойчивости векторных задач дискретной оптимизации ориентирован на получение результатов «качественного» характера, а именно, на выявление условий, при которых множество оптимальных (в определенном смысле) решений задачи обладает некоторым наперед заданным свойством инвариантности по отношению к внешним воздействиям на исходные данные задачи. Большинство результатов в этом направлении связано с получением необходимых и достаточных условий пяти известных типов устойчивости векторных целочисленных задач линейного и квадратичного программирования, состоящих в отыскании решений, оптимальных по Парето, Слейтеру, или Смейлу (см., например, [1-6]), а также булевых задач последовательной минимизации модулей линейных функций [7].

В настоящей работе проведен анализ пяти типов устойчивости (по векторному критерию) многокритериальной комбинаторной задачи по последовательно применяемым минимаксным критериям. В результате найдены необходимые и одновременно достаточные условия каждого типа устойчивости. Отметим, что аналогичные условия для векторной комбинаторной задачи на узкие места с паретовским принципом оптимальности были получены в [8].

\section{2. Определения, обозначения и свойства}

Рассмотрим векторный вариант известной комбинаторной задачи на узкие места. Пусть на множестве $N_{m}=\{1,2, \ldots, m\}, m \geqslant 2$, задана некоторая система непустых подмножеств $T \subseteq 2^{N_{m}} \backslash\{\varnothing\},|T| \geqslant 2$. Элементы множества $T$ принято называть траекториями. Пусть компонентами вектор-функции $f(t, A)=\left(f_{1}(t, A), f_{2}(t, A), \ldots, f_{n}(t, A)\right), n \geqslant 1$, заданной 
на $T$, являются минимаксные критерии

$$
f_{i}(t, A)=\max _{j \in t} a_{i j} \underset{t \in T}{\longrightarrow} \min , \quad i \in N_{n},
$$

где $A=\left[a_{i j}\right]$ - матрица размера $n \times m$ с элементами из $\mathbf{R}$.

Под векторной ( $n$-критериальной) комбинаторной задачей $Z^{n}(A), n \geqslant 1$, будем понимать задачу поиска лексикографического множества (множества лексикографических оптимумов)

$$
L^{n}(A)=\left\{t \in T: \forall t^{\prime} \in T\left(t \underset{A}{\succ} t^{\prime}\right)\right\},
$$

где $\succ_{A}-$ отрицание бинарного лексикографического отношения $\underset{A}{\succ}$, задаваемого на множестве траекторий $T$ формулой

$$
t \succ t^{\prime} \Longleftrightarrow \exists k \in N_{n}\left(f_{k}(t, A)>f_{k}\left(t^{\prime}, A\right) \& k=\min \left\{i \in N_{n}: f_{i}(t, A) \neq f_{i}\left(t^{\prime}, A\right)\right\}\right) .
$$

Отметим, что в схему скалярных (однокритериальных) комбинаторных задач (с линейными, минимаксными и другими критериями) вкладываются многие классические экстремальные задачи на графах (о коммивояжере, паросочетаниях, остовах и др.), разнообразные задачи теории расписаний и задачи булева программирования [1,9-11].

Будем использовать обозначение

$$
\overline{L^{n}}(A)=T \backslash L^{n}(A) .
$$

Свойство 1. Если $t \underset{A}{\succ} t^{\prime}$, то $t \in \overline{L^{n}}(A)$.

Свойство 2. Если $t \underset{A}{\succ} t^{\prime}$, mo $t^{\prime} \underset{A}{\succ} t$.

Известно (см., например, [12]), что задачу $Z^{n}(A)$ можно трактовать как задачу оптимизации по последовательно применяемым критериям, то есть лексикографическое множество $L^{n}(A)$ может быть определено как результат решения последовательности $n$ скалярных задач

$$
L_{i}^{n}(A)=\operatorname{Arg} \min \left\{f_{i}(t, A): t \in L_{i-1}^{n}(A)\right\}, \quad i \in N_{n},
$$

где $L_{0}^{n}(A)=T, \operatorname{Arg} \min \{\cdot\}$ - множество всех оптимальных траекторий соответствующей задачи минимизации. Отсюда вытекает справедливость соотношений

$$
T \supseteq L_{1}^{n}(A) \supseteq L_{2}^{n}(A) \supseteq \ldots \supseteq L_{n}^{n}(A)=L^{n}(A) .
$$

Очевидно, что при любой матрице $A \in \mathbf{R}^{n \times m}$ множество $L^{n}(A)$ непусто.

Понятно, что $t \succ_{A} t^{\prime}$, если $t \notin L_{k}^{n}(A)$ и $t^{\prime} \in L_{k}^{n}(A)$ при некотором индексе $k \in N_{n}$. Поэтому имеет место следующее свойство.

Свойство 3. Пусть индекс $k \in N_{n}$ таков, что $f_{k}(t, A)>f_{k}\left(t^{\prime}, A\right) u t^{\prime} \in L_{k}^{n}(A)$. Тогда $t \succ t^{\prime}$. A

Очевидно, что для любой траектории $t$ и всякой $n \times m$ матрицы $B$ с неотрицательными элементами $\left(B \in \mathbf{R}_{+}^{n \times m}\right)$ верны неравенства

$$
f_{i}(t, A) \leqslant f_{i}(t, A+B), \quad i \in N_{n} .
$$

Поэтому справедливо следующее свойство. 
Свойство 4. Пусть $k \in N_{n}$. Если траектория $t \in L_{k}^{n}(A)$ и матрица $B^{0} \in \mathbf{R}_{+}^{n \times m}$ таковы, что верны равенства $f_{i}(t, A)=f_{i}\left(t, A+B^{0}\right), i \in N_{k}$, то $t \in L_{k}^{n}\left(A+B^{0}\right)$.

Приведем определения пяти наиболее распространенных в литературе типов устойчивости (см., например, [1-8, 13-16].

Задача $Z^{n}(A), n \geqslant 1$, называется устойчивой (к возмущениям элементов матрицы $A$ ), если

$$
\exists \varepsilon>0 \quad \forall B \in \Xi(\varepsilon) \quad\left(L^{n}(A+B) \subseteq L^{n}(A)\right) .
$$

Здесь

$$
\Xi(\varepsilon)=\left\{B \in \mathbf{R}^{n \times m}:\|B\|<\varepsilon\right\}
$$

- множество возмущающих матриц,

$$
\|B\|=\max \left\{\left|b_{i j}\right|:(i, j) \in N_{n} \times N_{m}\right\},
$$

$B=\left[b_{i j}\right]$. Задачу $Z^{n}(A+B)$ будем в дальнейшем называть возмущенной.

Тем самым, задача устойчива, когда любые достаточно малые изменения исходных данных задачи не приводят к появлению новых лексикографических оптимумов. Поэтому устойчивость можно трактовать как дискретный аналог свойства полунепрерывности сверху по Хаусдорфу в точке $A$ многозначного (точечно-множественного) оптимального отображения

$$
L^{n}: \mathbf{R}^{n \times m} \rightarrow 2^{T}
$$

которое каждой матрице из $\mathbf{R}^{n \times m}$ ставит в соответствие лексикографическое множество задачи.

Отметим, что количественная оценка допустимых изменений исходных данных (так называемый радиус устойчивости) скалярной задачи на узкие места исследовалась в ряде работ (см., например, [10, 18, 19]).

Ослабляя требование непоявления новых лексикографических оптимумов, приходим к понятию сильной устойчивости задачи. Задача $Z^{n}(A)$ называется сильно устойчивой, если

$$
\exists \varepsilon>0 \quad \forall B \in \Xi(\varepsilon) \quad\left(L^{n}(A) \cap L^{n}(A+B) \neq \varnothing\right) .
$$

Тем самым, этот тип устойчивости описывает ситуацию, при которой лексикографическое множество задачи имеет непустое пересечение с аналогичным множеством любой из возмущенных задач. Легко видеть, что задача $Z^{n}(A)$ сильно устойчива, если она устойчива. Ниже будет показано (см. теорему 1), что верно и обратное утверждение.

Задача $Z^{n}(A)$ называется квазиустойчивой, если

$$
\exists \varepsilon>0 \quad \forall B \in \Xi(\varepsilon) \quad\left(L^{n}(A) \subseteq L^{n}(A+B)\right) .
$$

Иначе говоря, квазиустойчивость характеризует случай, когда все траектории лексикографического множества не теряют свойства оптимальности при достаточно малых возмущениях исходных данных задачи. Тем самым, квазиустойчивость задачи $Z^{n}(A)$ является дискретным аналогом свойства полунепрерывности снизу по Хаусдорфу в точке $A$ многозначного оптимального отображения (1).

Ослабляя требование сохранения всего лексикографического множества задачи, приходим к понятию сильной квазиустойчивости. Этот тип устойчивости связан с наличием хотя бы одной траектории, принадлежащей лексикографическим множествам всех 
возмущенных задач при небольших изменениях исходных данных. Итак, задача $Z^{n}(A)$ называется сильно квазиустойчивой, если

$$
\exists \varepsilon>0 \quad \exists t^{0} \in L^{n}(A) \quad \forall B \in \Xi(\varepsilon) \quad\left(t^{0} \in L^{n}(A+B)\right) .
$$

Очевидно, что всякая квазиустойчивая задача сильно квазиустойчива, а любая сильно квазиустойчивая задача является сильно устойчивой.

Наконец, задачу $Z^{n}(A)$ назовем стабильной, если

$$
\exists \varepsilon>0 \quad \forall B \in \Xi(\varepsilon) \quad\left(L^{n}(A)=L^{n}(A+B)\right) .
$$

Тем самым, свойство стабильности задачи $Z^{n}(A)$ является дискретным аналогом свойства непрерывности по Хаусдорфу в точке $A$ многозначного оптимального отображения (1). Очевидно, что задача $Z^{n}(A)$ стабильна в том и только том случае, когда она одновременно устойчива и квазиустойчива.

Отметим, что перечисленные выше типы устойчивости в работах [2-6] имеют другую терминологию ( $T_{1}-T_{5}$-устойчивость).

Для любого непустого подмножества $I \subseteq N_{n}$ на множестве траекторий $T$ задачи $Z^{n}(A)$ введем бинарные отношения:

$$
\begin{aligned}
& t \underset{I, A}{\geqslant} t^{\prime} \Longleftrightarrow \forall i \in I \quad\left(f_{i}(t, A) \geqslant f_{i}\left(t^{\prime}, A\right)\right), \\
& t_{I, A}^{>} t^{\prime} \Longleftrightarrow \forall i \in I \quad\left(f_{i}(t, A)>f_{i}\left(t^{\prime}, A\right)\right), \\
& t \underset{I, A}{\vdash} t^{\prime} \Longleftrightarrow \forall i \in I \quad\left(N_{i}(t, A) \supseteq N_{i}\left(t^{\prime}, A\right)\right),
\end{aligned}
$$

где

$$
N_{i}(t, A)=\left\{j \in t: a_{i j}=f_{i}(t, A)\right\} .
$$

Очевидны следующие свойства.

Свойство 5. Если $t \underset{I, A}{\vdash} t^{\prime}$, то существует такое число $\varepsilon>0$, что для любой возмущчающеей матрицы $B \in \Xi(\varepsilon)$ выполняется отношение $t \underset{I, A+B}{\geqslant} t^{\prime}$.

Свойство 6. Если $t \underset{N_{n}, A}{\geqslant} t^{\prime}$, mо $t^{\prime} \underset{A}{\succ} t$.

Учитывая свойства 5 и 6 , а также непрерывность функции $f(t, A)$ на множестве параметров $\mathbf{R}^{n \times m}$, убеждаемся в справедливости следующих двух свойств.

Свойство 7. Если $t \underset{N_{n}, A}{\vdash} t^{\prime}$, то

$$
\exists \varepsilon>0 \quad \forall B \in \Xi(\varepsilon) \quad\left(t^{\prime} \underset{A+B}{\succ} t\right) .
$$

Свойство 8. Если для траекторий $t$ и $t^{\prime}$ выполняется любое из условий:

(i) $t \underset{1, A}{>} t^{\prime}$,

(ii) $\exists k \in N_{n-1}\left(t \underset{N_{k}, A}{\vdash} t^{\prime} \& t \underset{k+1, A}{>} t^{\prime}\right)$,

то справедлива формула

$$
\exists \varepsilon>0 \quad \forall B \in \Xi(\varepsilon) \quad\left(t \underset{A+B}{\succ} t^{\prime}\right) .
$$


Далее положим

$$
\begin{aligned}
M(t) & =\left\{i \in N_{n}: t \in L_{i}^{n}(A)\right\}, \\
\sigma(t) & =|M(t)|, \\
V^{n}(A) & =\left\{t \in L_{1}^{n}(A): \exists t^{\prime} \in L^{n}(A)\left(t \underset{M(t), A}{\vdash} t^{\prime}\right)\right\}, \\
U^{n}(A) & =\left\{t \in L^{n}(A): \forall i \in N_{n} \forall t^{\prime} \in L_{i}^{n}(A)\left(t^{\prime} \vdash i, A\right)\right\} .
\end{aligned}
$$

Очевидно, что множество $U^{n}(A)$ может быть любым (в том числе и пустым) подмножеством лексикографического множества $L^{n}(A)$.

В силу рефлексивности отношения $\underset{i, A}{\vdash}$, справедливо включение $L^{n}(A) \subseteq V^{n}(A)$, и поэтому $L^{n}(A)=V^{n}(A)$, если $L^{n}(A)=L_{1}^{n}(A)$. Также легко видеть, что при $t \in L_{1}^{n}(A)$ множество $M(t)$ совпадает с множеством $N_{\sigma(t)}$, где $1 \leqslant \sigma(t) \leqslant n$. Причем $\sigma(t)=n$ лишь в случае, когда $t \in L^{n}(A)$. Поэтому имеет место следующее свойство.

Свойство 9. Пусть $t \in L_{1}^{n}(A) \backslash L^{n}(A) u t^{\prime} \in L^{n}(A)$. Тогда $1 \leqslant \sigma(t)<n u t \underset{\sigma(t)+1, A}{>} t^{\prime}$.

Легко также видеть, что справедливы следующие свойства.

Свойство 10. Если $t \in L_{1}^{n}(A) \backslash V^{n}(A)$, то $N_{m} \backslash t \neq \varnothing$.

Свойство 11. Если $t \in L^{n}(A) u t^{\prime} \in U^{n}(A)$, mо $t \underset{N_{n}, A}{\vdash} t^{\prime}$.

\section{3. Вспомогательные утверждения}

Лемма 1. Если $t \in L_{1}^{n}(A) \backslash V^{n}(A)$, то

$$
\forall \varepsilon>0 \quad \exists B^{0} \in \Xi(\varepsilon) \quad \forall t^{\prime} \in L^{n}(A) \quad\left(t^{\prime} \underset{A+B^{0}}{\succ} t\right) .
$$

Доказательство. Пусть $t \in L_{1}^{n}(A) \backslash V^{n}(A)$. Тогда для любой траектории $t^{\prime} \in L^{n}(A)$ существует такой индекс $k=k\left(t^{\prime}\right) \in M(t)=N_{\sigma(t)}$, что $N_{k}(t, A) \nsupseteq N_{k}\left(t^{\prime}, A\right)$ и $t \in L_{k}^{n}(A)$. Тогда $t^{\prime} \backslash t \neq \varnothing$. Пусть $p \in N_{k}\left(t^{\prime}, A\right) \backslash N_{k}(t, A)$. Тогда

$$
f_{k}(t, A)=f_{k}\left(t^{\prime}, A\right)=a_{k p} .
$$

Далее, положив $\varepsilon>0$ и учитывая свойство 10, построим возмущающую матрицу $B^{0}=\left[b_{i j}^{0}\right] \in \mathbf{R}^{n \times m}$ по правилу

$$
b_{i j}^{0}= \begin{cases}\delta, & \text { если } i \in M(t), j \in N_{m} \backslash t, \\ 0 & \text { в остальных случаях, }\end{cases}
$$

где $0<\delta<\varepsilon$. Тогда очевидны равенства

$$
f_{i}(t, A)=f_{i}\left(t, A+B^{0}\right), \quad i \in M(t) .
$$

Поэтому справедливо включение $t \in L_{k}^{n}\left(A+B^{0}\right)$ в силу свойства 4 и включения $t \in L_{k}^{n}(A)$. Кроме того, используя равенства (4), получаем, что

$$
\begin{aligned}
f_{k}\left(t^{\prime}, A+B^{0}\right) & =\max \left\{a_{k j}+b_{k j}^{0}: j \in t^{\prime}\right\}=\max \left\{a_{k j}+b_{k j}^{0}: j \in t^{\prime} \backslash t\right\} \\
& =a_{k p}+\delta>a_{k p}=f_{k}\left(t^{\prime}, A\right)=f_{k}(t, A)=f_{k}\left(t, A+B^{0}\right) .
\end{aligned}
$$

Из свойства 3 получаем формулу (3). Лемма 1 доказана. 
Лемма 2. Если $L_{1}^{n}(A)=V^{n}(A) u t \in \overline{L^{n}}(A)$, то найдется такая траектория $t^{0} \in L^{n}(A)$, что

$$
\exists \varepsilon>0 \quad \forall B \in \Xi(\varepsilon) \quad\left(t \underset{A+B}{\succ} t^{0}\right) .
$$

Доказательство. Для траектории $t \in \overline{L^{n}}(A)$ рассмотрим два возможных случая.

Случай 1: $t \in L_{1}^{n}(A)$. Тогда $t \in V^{n}(A)$ и потому существует такая траектория $t^{0} \in L^{n}(A)$, что выполняется отношение $t \underset{M(t), A}{\vdash} t^{0}$. Кроме того, с учетом включения $t \in \overline{L^{n}}(A) \cap L_{1}^{n}(A)=L_{1}^{n}(A) \backslash L^{n}(A)$ и свойства 9 заключаем, что $t \underset{\sigma(t)+1, A}{>} t^{0}$. Отсюда и
из свойства 8 получаем, что

$$
\exists \varepsilon_{1}>0 \quad \forall B \in \Xi\left(\varepsilon_{1}\right) \quad\left(t \underset{A+B}{\succ} t^{0}\right) .
$$

Случай 2: $t \in T \backslash L_{1}^{n}(A)$. Тогда для любой траектории $t^{0} \in L^{n}(A)$ выполняется отношение $t \underset{1, A}{>} t^{0}$. Поэтому из свойства 8 вытекает, что

$$
\exists \varepsilon_{2}>0 \quad \forall B \in \Xi\left(\varepsilon_{2}\right) \quad\left(t \underset{A+B}{\succ} t^{0}\right) .
$$

Учитывая оба случая, убеждаемся в справедливости формулы (5). Лемма 2 доказана.

Лемма 3. Если $t \in U^{n}(A) u t^{\prime} \in T$, то

$$
\exists \varepsilon>0 \quad \forall B \in \Xi(\varepsilon) \quad\left(t \underset{A+B}{\succ} t^{\prime}\right) .
$$

Доказательство. Пусть $t \in U^{n}(A)$. Для траектории $t^{\prime}$ рассмотрим два возможных случая.

Случай 1: $t^{\prime} \in L_{1}^{n}(A)$. Пусть сначала $t^{\prime} \in L^{n}(A)$. Тогда в силу свойства 11 справедливо отношение $t^{\prime} \underset{N_{n}, A}{\vdash} t$. Отсюда, используя свойство 7, получаем (6).

Пусть $t^{\prime} \in L_{1}^{n}(A) \backslash L^{n}(A)$. Тогда существует такой индекс $k=k\left(t^{\prime}\right) \in N_{n} \backslash\{1\}$, что $t^{\prime} \notin L_{k}^{n}(A)$, а $t^{\prime} \in L_{i}^{n}(A)$ при $i \in N_{k-1}$. Поэтому имеют место отношения $t^{\prime} \underset{N_{k-1}, A}{\vdash} t$ и $t^{\prime} \underset{k, A}{>} t$. Пользуясь этими фактами и свойством 8 , заключаем, что верна формула

$$
\exists \varepsilon>0 \quad \forall B \in \Xi(\varepsilon) \quad\left(t^{\prime} \underset{A+B}{\succ} t\right),
$$

которая, ввиду свойства 2, дает (6).

Случай 2: $t^{\prime} \in T \backslash L_{1}^{n}(A)$. Тогда имеет место отношение $t^{\prime}>t$. Отсюда и из свойств 2 и 8 вытекает формула (6).

Лемма 3 доказана.

Лемма 4. Если $t \in L^{n}(A) \backslash U^{n}(A)$, то верна формула

$$
\exists t^{0} \in T \quad \forall \varepsilon>0 \quad \exists B^{0} \in \Xi(\varepsilon) \quad\left(t \underset{A+B^{0}}{\succ} t^{0}\right) .
$$

Доказательство. Поскольку $t \notin U^{n}(A)$, существуют $k \in N_{n}$ и $t^{0} \in L_{k}^{n}(A)$ такие, что $N_{k}\left(t^{0}, A\right) \nsupseteq N_{k}(t, A)$ и $t \in L_{k}^{n}(A)$. Пусть $p \in N_{k}(t, A) \backslash N_{k}\left(t^{0}, A\right)$. Тогда

$$
f_{k}(t, A)=f_{k}\left(t^{0}, A\right)=a_{k p}
$$


Поэтому, положив $\varepsilon>0$ и построив элементы возмущающей матрицы $B^{0}=\left[b_{i j}^{0}\right] \in \mathbf{R}^{n \times m}$ по правилу

$$
b_{i j}^{0}= \begin{cases}\delta, & \text { если } i=k, j=p, \\ 0 & \text { в остальных случаях, }\end{cases}
$$

где $0<\delta<\varepsilon$, убеждаемся в справедливости соотношений

$$
\begin{aligned}
f_{i}\left(t^{0}, A\right) & =f_{i}\left(t^{0}, A+B^{0}\right), \quad i \in N_{k}, \\
f_{k}\left(t, A+B^{0}\right) & =\max \left\{a_{k j}+b_{k j}^{0}: j \in t\right\}=a_{k p}+\delta>a_{k p}=f_{k}(t, A)=f_{k}\left(t^{0}, A\right) \\
& =f_{k}\left(t^{0}, A+B^{0}\right) .
\end{aligned}
$$

Отсюда следует, что $t^{0} \in L_{k}^{n}\left(A+B^{0}\right)$ согласно свойству 4 и $t \underset{A+B^{0}}{\succ} t^{0}$ согласно свойству 3 . Следовательно, верна формула (7). Лемма 4 доказана.

\section{4. Анализ пяти типов устойчивости}

Теорема 1. Для векторной задачи $Z^{n}(A), n \geqslant 1$, следующие утверждения эквивалентны:

(1) задача $Z^{n}(A)$ устойчива,

(2) задача $Z^{n}(A)$ сильно устойчива,

(3) $L_{1}^{n}(A)=V^{n}(A)$.

Доказательство. Импликация (1) $\Rightarrow$ (2) очевидна.

Докажем, что $(2) \Rightarrow(3)$. Допустим противное, то есть задача $Z^{n}(A)$ сильно устойчива, но $L_{1}^{n}(A) \neq V^{n}(A)$. Пусть $t \in L_{1}^{n}(A) \backslash V^{n}(A)$. Тогда по лемме 1 , ввиду свойства 1 ,

$$
\forall \varepsilon>0 \quad \exists B^{0} \in \Xi(\varepsilon) \quad \forall t^{\prime} \in L^{n}(A) \quad\left(t^{\prime} \in \overline{L^{n}}\left(A+B^{0}\right)\right) .
$$

Отсюда получаем, что

$$
\forall \varepsilon>0 \quad \exists B^{0} \in \Xi(\varepsilon) \quad\left(L^{n}(A) \cap L^{n}\left(A+B^{0}\right)=\varnothing\right),
$$

что противоречит сильной устойчивости задачи $Z^{n}(A)$.

Докажем, что (3) $\Rightarrow(1)$. Очевидно, что при выполнении равенства $L^{n}(A)=T$ задача $Z^{n}(A)$ устойчива. Пусть $\overline{L^{n}}(A) \neq \varnothing$. Тогда, в силу леммы 2 и свойства 1 , для всякой траектории $t \in \overline{L^{n}}(A)$ находим, что

$$
\exists \varepsilon=\varepsilon(t)>0 \quad \forall B \in \Xi(\varepsilon) \quad\left(t \in \overline{L^{n}}(A+B)\right) .
$$

Поэтому при

$$
\varepsilon^{*}=\min \left\{\varepsilon(t): t \in \overline{L^{n}}(A)\right\}
$$

легко видеть, что

$$
\exists \varepsilon^{*}>0 \quad \forall B \in \Xi\left(\varepsilon^{*}\right) \quad\left(\overline{L^{n}}(A) \subseteq \overline{L^{n}}(A+B)\right) .
$$

Следовательно, задача $Z^{n}(A)$ устойчива. Теорема 1 доказана. 
Отметим, что установленная теоремой 1 эквивалентность понятий устойчивости и сильной устойчивости задачи $Z^{n}(A)$ не всегда присуща векторной задаче дискретной оптимизации. Например, в [17] показано, что всякая векторная задача целочисленного линейного программирования с паретовским принципом оптимальности сильно устойчива, хотя давно известно (см., например, $[1,2])$, что такая задача устойчива тогда и только тогда, когда совпадают множества Парето и Слейтера.

Теорема 1 имеет следующую эквивалентную формулировку.

Теорема 2. Задача $Z^{n}(A)$ устойчива (сильно устойчива) тогда и только тогда, когда справедлива импликаиия

$$
L^{n}(A) \neq L_{1}^{n}(A) \Longrightarrow \forall t \in L_{1}^{n}(A) \backslash L^{n}(A) \quad \exists t^{0} \in L^{n}(A) \quad\left(t \underset{M(t), A}{\vdash} t^{0}\right) .
$$

Отсюда вытекает следующее утверждение.

Следствие 1. При выполнении любого из условий

(1) $L^{n}(A)=L_{1}^{n}(A)$,

(2) $\left|\left\{y \in \mathbf{R}^{n}: y=f(t, A), t \in L_{1}^{n}(A)\right\}\right|=1$,

(3) $\forall t \in \overline{L^{n}}(A) \forall t^{\prime} \in L^{n}(A)\left(t \underset{1, A}{>} t^{\prime}\right)$,

(4) $\forall t \in \overline{L^{n}}(A) \exists t^{\prime} \in T\left(t \underset{1, A}{>} t^{\prime}\right)$

задача $Z^{n}($ A) устойчива и сильно устойчива.

Следующий пример показывает, что равенство $L^{n}(A)=L_{1}^{n}(A)$ не является необходимым условием устойчивости (сильной устойчивости).

Пример 1. Пусть

$$
n=2, \quad m=2, \quad T=\left\{t_{1}, t_{2}\right\}, \quad t_{1}=\{1\}, \quad t_{2}=\{1,2\}, \quad A=\left(\begin{array}{ll}
1 & 1 \\
1 & 2
\end{array}\right) .
$$

Тогда

$$
f\left(t_{1}, A\right)=(1,1), \quad f\left(t_{2}, A\right)=(1,2), \quad L^{2}(A)=\left\{t_{1}\right\}, \quad L_{1}^{2}(A)=T .
$$

Легко видеть, что

$$
M\left(t_{1}\right)=\{1,2\}, \quad M\left(t_{2}\right)=\{1\}, \quad t_{1} \underset{M\left(t_{1}\right), A}{\vdash} t_{1}, \quad t_{2} \underset{M\left(t_{2}\right), A}{\vdash} t_{1} .
$$

Поэтому

$$
V^{2}(A)=L_{1}^{2}(A)
$$

Следовательно, согласно теореме 1 , задача $Z^{2}(A)$ устойчива, хотя $L^{2}(A) \neq L_{1}^{2}(A)$.

Отметим, что в работах [13-15] показано, что приведенные в следствии 1 условия являются не только достаточными, но и необходимыми для устойчивости и сильной устойчивости векторной комбинаторной задачи, если критерии являются линейными.

В скалярном случае $(n=1)$ теорема 1 превращается в следующее утверждение. 
Следствие 2. Скалярная задача $Z^{1}(A)$ устойчива и сильно устойчива при любом векторе $A \in \mathbf{R}^{m}$.

Теорема 3. Векторная задача $Z^{n}(A), n \geqslant 1$, квазиустойчива тогда и только тогда, когда $L^{n}(A)=U^{n}(A)$.

Доказательство. Докажем необходимость. Пусть, напротив, задача $Z^{n}(A)$ квазиустойчива, но $L^{n}(A) \neq U^{n}(A)$. Тогда существует траектория $t \in L^{n}(A) \backslash U^{n}(A)$, для которой, в силу леммы 4 и свойства 1 , справедлива формула

$$
\forall \varepsilon>0 \quad \exists B^{0} \in \Xi(\varepsilon) \quad\left(t \in \overline{L^{n}}\left(A+B^{0}\right)\right) .
$$

Отсюда выводим, что

$$
\forall \varepsilon>0 \quad \exists B^{0} \in \Xi(\varepsilon) \quad\left(L^{n}(A) \nsubseteq L^{n}\left(A+B^{0}\right)\right),
$$

что противоречит квазиустойчивости задачи $Z^{n}(A)$.

Докажем достаточность. Пусть $L^{n}(A)=U^{n}(A)$, и пусть $t \in U^{n}(A)$. Тогда для любой траектории $t^{\prime} \in T$, в силу леммы 3 , справедлива формула

$$
\exists \varepsilon\left(t^{\prime}\right)>0 \quad \forall B \in \Xi\left(\varepsilon\left(t^{\prime}\right)\right) \quad\left(t \underset{A+B}{\succ} t^{\prime}\right) .
$$

Таким образом, для всякой траектории $t \in L^{n}(A)$ существует такое $\varepsilon=\varepsilon(t)>0$, что при всякой возмущающей матрице $B \in \Xi(\varepsilon)$ выполнено включение $t \in L^{n}(A+B)$. Поэтому, полагая

$$
\varepsilon^{*}=\min \left\{\varepsilon(t): t \in L^{n}(A)\right\},
$$

получаем, что

$$
\exists \varepsilon^{*}>0 \quad \forall B \in \Xi\left(\varepsilon^{*}\right) \quad\left(L^{n}(A) \subseteq L^{n}(A+B)\right) .
$$

Следовательно, задача $Z^{n}(A)$ квазиустойчива. Теорема 2 доказана.

Очевидна следующая эквивалентная формулировка теоремы 3.

Теорема 4. Для того чтобы векторная задача $Z^{n}(A)$ была квазиустойчива, необходимо и достаточно, чтобы выполнялась формула

$$
\forall t \in L^{n}(A) \quad \forall i \in N_{n} \quad \forall t^{\prime} \in L_{i}^{n}(A) \quad\left(t^{\prime} \underset{i, A}{\vdash} t\right)
$$

Следствие 3. Скалярная задача $Z^{1}(A), A \in \mathbf{R}^{m}$, квазиустойчива тогда и только тогда, когда

$$
\forall t, t^{\prime} \in L^{1}(A) \quad\left(N_{1}(t, A)=N_{1}\left(t^{\prime}, A\right)\right) .
$$

Здесь $L^{1}(A)$ - множество оптимальных траекторий скалярной задачи $Z^{1}(A)$.

Теорема 5. Векторная задача $Z^{n}(A), n \geqslant 1$, сильно квазиустойчива тогда и только тогда, когда $U^{n}(A) \neq \varnothing$.

Доказательство. Докажем необходимость. Пусть $U^{n}(A)=\varnothing$. Тогда для любой траектории $t \in L^{n}(A)$, согласно лемме 4 и свойству 1 ,

$$
\forall \varepsilon>0 \quad \exists B^{0} \in \Xi(\varepsilon) \quad\left(t \in \overline{L^{n}}\left(A+B^{0}\right)\right) .
$$


Поэтому задача $Z^{n}(A)$ не является сильно квазиустойчивой.

Докажем достаточность. Пусть $t \in U^{n}(A)$. Тогда, по лемме 3, для любой траектории $t^{\prime} \in T$ верна формула

$$
\exists \varepsilon=\varepsilon\left(t^{\prime}\right)>0 \quad \forall B \in \Xi(\varepsilon) \quad\left(t \underset{A+B}{\succ} t^{\prime}\right)
$$

Отсюда, согласно определению лексикографического множества,

$$
\exists \varepsilon^{*}>0 \quad \forall B \in \Xi\left(\varepsilon^{*}\right) \quad\left(t \in L^{n}(A+B)\right),
$$

где

$$
\varepsilon^{*}=\min \left\{\varepsilon\left(t^{\prime}\right): t^{\prime} \in T\right\}
$$

Следовательно, задача $Z^{n}(A)$ сильно квазиустойчива. Теорема 5 доказана.

Следствие 4. Скалярная задача $Z^{1}(A)$ сильно квазиустойчива тогда и только тогда, когда

$$
\exists t^{0} \in L^{1}(A) \quad \forall t \in L^{1}(A) \quad\left(t \underset{1, A}{\vdash} t^{0}\right) .
$$

Непосредственно из теорем 1 и 3 вытекает следующее утверждение.

Теорема 6. Задача $Z^{n}(A), n \geqslant 1$, стабильна тогда и только тогда, когда

$$
V^{n}(A)=L_{1}^{n}(A), \quad U^{n}(A)=L^{n}(A) .
$$

Из теорем 1-6 вытекает следующее утверждение.

Следствие 5. Если $\left|L_{1}^{n}(A)\right|=1$, то задача $Z^{n}(A)$ является одновременно устойчивой, сильно устойчивой, квазиустойчивой, сильно квазиустойчивой и стабильной.

\section{Список литературы}

1. Сергиенко И. В., Козерацкая Л. Н., Лебедева Т. Т., Исследование устойчивости и параметрический анализ дискретных оптимизационных задач. Наукова думка, Киев, 1995.

2. Сергиенко И. В., Шило В. П., Задачи дискретной оптимизачии. Проблемы, методы ремения, исследования. Наукова думка, Киев, 2003.

3. Лебедева Т. Т., Сергиенко Т. И., Сравнительный анализ различных типов устойчивости по ограничениям векторной задачи целочисленной оптимизации. Кибернетика и системный анализ (2004), № 1, 63-70.

4. Лебедева Т. Т., Семенова Н. В., Сергиенко Т. И., Устойчивость векторных задач целочисленной оптимизации: взаимосвязь с устойчивостью множеств оптимальных и неоптимальных решений. Кибернетика и системный анализ (2005), № 4, 90-100.

5. Лебедева Т. Т., Сергиенко Т. И., Устойчивость по векторному критерию и ограничениям векторной целочисленной задачи квадратичного программирования. Кибернетика и системный анализ (2006), № 5, 63-72.

6. Лебедева Т. Т., Сергиенко Т. И., Разные типы устойчивости векторной задачи целочисленной оптимизации: общий подход. Кибернетика и системный анализ (2008), № 3, 142-148.

7. Емеличев В. А., Гуревский Е. Е., Булева задача последовательной минимизации модулей линейных функций и теоремы устойчивости. Кибернетика и системный анализ (2007), № 5, 178-187. 
8. Емеличев В. А., Кузьмин К. Г., Критерии устойчивости векторных комбинаторных задач “на узкие места" в терминах бинарных отношений. Кибернетика и системный анализ (2008), № 3 , 103-111.

9. Емеличев В. А., Кравцов М. К., О комбинаторных задачах векторной оптимизации. Дискретная математика (1995) 7, №1, 3-18.

10. Sotskov Yu. N., Leontev V. K., Gordeev E. N., Some concepts of stability analysis in combinatorial optimization. Discrete Appl. Math. (1995) 58, №2, 169-190.

11. Сотсков Ю. Н., Сотскова Н. Ю., Теория расписаний. Системы с неопределенными числовыми параметрами. ОИПИ НАН Беларуси, Минск, 2004.

12. Подиновский В. В., Гаврилов В. М., Оптимизация по последовательно применяемым критериям. Советское радио, Москва, 1975.

13. Емеличев В. А., Бердышева Р. А., О радиусах устойчивости, квазиустойчивости и стабильности векторной траекторной задачи лексикографической оптимизации. Дискретная математика (1998) 10, №1, 20-27.

14. Емеличев В. А., Бердышева Р. А., О сильной устойчивости векторной траекторной задачи лексикографической оптимизации. Дискретная математика (1998) 10, №3, 3-9.

15. Бердышева Р. А., Емеличев В. А., Некоторые виды устойчивости комбинаторной задачи лексикографической оптимизации. Известия вузов. Математика (1998), № 12, 11-21.

16. Emelichev V. A., Girlich E., Nikulin Yu. V., Podkopaev D. P., Stability and regularization of vector problems of integer linear programming. Optimization (2002) 51, №4, 645-676.

17. Emelichev V. A., Nikulin Y. V., Numerical measure of strong stability and strong quasi-stability in the vector problem of integer linear programming. Comput. Sci. J. Mold. (1999) 7, №1, 105-117.

18. Гордеев Э. Н., Об устойчивости задач на узкие места. Журнал вычисл. матем. и матем. физики (1993) 33, №9, 1391-1402.

19. Гордеев Э. Н., Леонтьев В. К., Общий подход к исследованию устойчивости решений в задачах дискретной оптимизации. Журнал вычисл. матем. и матем. физики (1996) 36, №1, 66-72.

Статья поступила 28.08.2008. 